\title{
Research on Design of Video Teaching System of College English Course based on Computer Intelligence Algorithm
}

\author{
Jing Liao \\ Wuhan Business University, Wuhan, Hubei, 430056
}

Keywords: video teaching; College English Course; computer intelligence

\begin{abstract}
With the rapid development of network technology and computer technology, the comprehensive development of multimedia technology and network technology has become a trend. In order to meet the current needs of English teaching, this paper proposes an English teaching system based on MVC architecture, and analyzes its function design and architecture layout in detail. At the same time, a streaming media server is set up to use a third-party ActiveX space to implement web page video playback. Finally, the system is tested and each function is well-functioning.
\end{abstract}

\section{Introduction}

"Microteaching" has been widely used in teaching in countries all over the world. It divides teaching skills into various micro-skills. Weinberger defines these micro-contents as "unit blocks, loosely connected, information units that are always in a dynamic reorganization." Reina expressed micro-learning as "a new type of learning based on micro-content and micro-media that exists in the new media ecosystem." Micro-curriculum is a form of curriculum that consists of a series of semi-independent units (topics) within the scope of a discipline. It is based on teachers' and students' interests and creates activity designs and scenarios that emphasize depth but not breadth. Micro-curriculum is not simply to integrate technology into the curriculum. It is often based on a learning theme to organize relevant teaching activities. The micro-curricular must have a light and even entertaining learning experience in a relaxed state of mind. This kind of micro-curriculum can be a "nutritious fast food" and can satisfy students' tastes. Obviously, the construction of digital micro-content resources with sufficient sources and high performance is the basis for micro-learning. However, at present, the field still lacks resources that meet the requirements, and in particular, it meets the needs of personalized, dynamic and visually penetrating micro-course content and its structure. Worth exploring. The school-based curriculum of college English has a long span of time. Students tend to fall into the state of knowledge-fatiguing in their studies. The design of the mini-video curriculum exactly meets the habit of students who love movies and television content. By linking the video content of a good instructional design and adding quality control measures, it not only caters to students' tastes, but also stimulates students' interest and potential in learning. However, there are few micro-multimedia curriculum resources for college English. How to reorganize the school-based resources into a micro-multimedia courseware library, combined with teaching reform experience, believes that reconstructing college English video resources is a micro-curriculum resource that makes up for student time and fragmentation, in line with its love of British and American film and television, To learn a short-lived common habit of enthusiasm, as long as the production of a reasonable college English web video mini courseware template, and rebuild its production process, you can solve this problem.

\section{Teaching Design of College English WEB Video Mini Course}

Language learning is a kind of cultural influence. It cannot rely on the limited time of the classroom to improve its communication skills. Promoting the miniaturization of college English courses and increasing the diversity, flexibility, and adaptability to the development needs of different students become feasible and effective teaching design strategies. Establish a teaching 
design model centering on "learning", emphasizing learners as the center, creating learning situations, analyzing difficult points, asking questions, decomposing cases, providing learners with rich learning strategies, and using learners' learning processes. Initiative and constructivism. Starting from this guiding principle, the teaching model of college English micro video teaching courses is established in view of college students' inclination to acquire knowledge from television and movies.

The teaching model consists of two parts: the reorganization of the content of video teaching clips and the control and analysis of teaching quality. The reorganization of the content of video teaching clips consists of three parts: First, the topical video content is cut into pieces and converted into a streaming media format; then, a number of difficulties and doubts are decomposed and produced in a variety of objective questions. Online quizzes can automatically give results and feedback; finally, background knowledge is designed through the context to allow students to understand the main content of the video. The content of the reorganization is short and refined, with a prominent theme. In the teaching quality control and analysis module, the teaching objectives of the micro video course are decomposed and clarified, and only small targets that need innovation are set, for example, the students are required to complete the performance of the role simulation show. Difficulties and doubts in the Mini-course were completed in the form of PPT, then converted to FLASH animation using ISPRINGPRESENTER, and Quiz Creator created the quiz to complete students' mastery of difficulties and doubts. Ask questions for interactive teaching. The sound simulation is to separate the sound files in the video, and then through the plug-in technology to allow students to simulate the role of the microphone training, the student's sound waveform and the audio waveform in the video to judge, give the fit between the two To test the results of spoken language training in the process of role play. After the class, organize a character performance in the video, let the students direct themselves, organize themselves, join the plots, and compile their own recordings to improve the team collaboration ability and organizational development ability among the students. Turn the boring student process into a game. Entertainment experience learning process.

\section{Template Design of College English WEB Video Mini Course}

The learning objectives of micro-curricular courses, the knowledge content of content blocks, the learning time, and the attitude of students to learning have all exhibited the characteristics of "miniature" and "small". Therefore, it was decided that the template structure of micro-courses would not be like the structure of ordinary courses. complete. The successful experience of the Singapore Minicourse has shown that there are five different teaching modes for design and development: problem-based learning; case-based learning; resource-based learning; scenario-based learning and collaborative learning. From the point of view of the special course of college English, it is not only a compulsory course, but also a language communication ability. Therefore, the design of college English micro video curriculum incorporates elements of problem-based learning, resources, situational learning, and collaborative learning in the Singaporean teaching model. Experience shows that a 6-minute college English WEB video mini-course, where the main material content takes up $60 \%$ of the time, the remaining $40 \%$ of the time is specifically allocated as shown in Table 1. Therefore, we must first consider the time constraints in the instructional design. In the later non-linear editing, we can adjust the time period of the film. The whole process constitutes a small cycle of the knowledge module.

\section{Overall Architecture Design of System}

System architecture as the basis for system operation, its layout will determine the stability of its operation. In the J2EE architecture, server-side web applications can be divided into several different layers. Each of these different layers has different functions and links with different communication interfaces.

Among them, the JSP page serves as the view layer, which is mainly used for the interaction 
between the system and the user, and the final result is displayed directly through the JSP page. View It cannot perform actual business but can accept data updates, making the system interface up-to-date. The implementation of the page is achieved through the JSP language. Action control layer, which mainly receives response requests from client users, and then invokes the model in its business layer to implement the transfer of user requests. When the user submits the relevant request through the page, it sends an html form, and the controller responds to its request, calls the relevant model to process it, and finally passes the result to the user through an attempt. In this layer, the controller is implemented by Action Servlet and Action. Among them, the Action Servlet is mainly responsible for receiving the HTTP response in the previous section, and forwards the relevant request to the corresponding struts-config.xml information. Action class. In this layer, the Action can be seen as an adapter, separating the request from the business logic so that the user and the user's own needs can invoke the corresponding business logic components. Action is mainly used to control the flow of the application program. The Service business layer is between the Action layer and the persistence layer. The main function of this layer is usually the processing of application logic services and verification services, such as login password verification and interface connection. This layer usually divides the function into different steps and is achieved by invoking the underlying DAO layer. The DAO layer is mainly used to connect to a database and perform related operations on the database, such as adding, deleting, modifying, and querying related business functions. In J2EE development, queries are usually implemented through SQL statements. To achieve access to the database, then call each of the interfaces, you can achieve.

\section{System Function Realization}

The specific development environment of the system is: Operating System: Win7 Database: Mysql 5.0 Server: Tomcat6.5 Development Platform: MyEclipse3.5 Development Technology: Struts2, Hibernate3, Spring2

This article selects Real Network Helix Serve as a streaming media server that has streaming media servers supporting multiple formats and cross-platform. And the server can also support some current mainstream playback software, such as Apple QuickTime, Windows Media Playe and so on. In this article, we need to realize how the target is inserted into the broadcaster through the interface and realize the playback of English audio/video materials. In this regard, the article selected ActiveX control technology in the development. The principle of this technology is to insert ActiveX controls in the HTML file via objet object tags, and to implement access to this space through Javascript among them. The user only needs to install the plug-in, and the interface realizes the playing of English materials. The playback code is: <script language="javascript" $>$ function play(filename) \{top.document.all("iVideo").src="video.jsp?src="+filename; $\}</$ script $>$

Action control layer, which mainly receives response requests from client users, and then invokes the model in its business layer to implement the transfer of user requests. When the user submits the relevant request through the page, it sends an html form, and the controller responds to its request, calls the relevant model to process it, and finally passes the result to the user through an attempt. In this layer, the controller is implemented by Action Servlet and Action. Among them, the Action Servlet is mainly responsible for receiving the HTTP response in the previous section, and forwards the relevant request to the corresponding struts-config.xml information. Action class. In this layer, the Action can be seen as an adapter, separating the request from the business logic so that the user and the user's own needs can invoke the corresponding business logic components. Action is mainly used to control the flow of the application program.

\section{Conclusion}

In this article, the current popular streaming media technology adopts third-party controls to implement the playback of related videos, so that students can improve their oral English and listening skills through the system and can better improve their English performance. English teaching has a great reference. 


\section{References}

[1] Wang Yan, Tang Fei. China's boutique hotel development conditions analysis [J]. Journal of Changchun University of Science and Technology (Comprehensive Edition), 2006, (4): 40.

[2] Zheng Xiangmin. Explore the development of Chinese boutique hotels [J]. Hotel Modernization, 2006, (6): 39.

[3] Li Yingjun. Experienced economy era hotel marketing strategy [J]. Economic Forum, 2007, (7).

[4] Zhang Weiyu. The feasibility study of developing boutique hotels in Sichuan [J]. National Business (Economic Theory Research), 2009, (14).

[5] Fang Yongxiang. Research on Design of Guidance Model Based on Microclass[J]. Teaching and Management: Theory, 2015, 2(06):106-109. 